\title{
Norois
}

Environnement, aménagement, société

$228 \mid 2013$

La patrimonialisation des paysages de l'eau dans

l'ouest de la France

\section{Les épis de la Loire armoricaine, un héritage à la patrimonialité incertaine}

Groynes of the Armorican Lower Loire River, an Heritage with Uncertain Future

Régis Barraud, Nathalie Carcaud, Hervé Davodeau, David Montembault et Claire Pordoy

\section{OpenEdition}

\section{Journals}

Édition électronique

URL : https://journals.openedition.org/norois/4729

DOI : 10.4000 /norois.4729

ISBN : 978-2-7535-2918-2

ISSN : $1760-8546$

\section{Éditeur}

Presses universitaires de Rennes

\section{Édition imprimée}

Date de publication : 30 septembre 2013

Pagination : $39-52$

ISBN : 978-2-7535-2883-3

ISSN : 0029-182X

\section{Référence électronique}

Régis Barraud, Nathalie Carcaud, Hervé Davodeau, David Montembault et Claire Pordoy, «Les épis de la Loire armoricaine, un héritage à la patrimonialité incertaine », Norois [En ligne], 228 | 2013, mis en ligne le 30 septembre 2015, consulté le 13 janvier 2022. URL : http://journals.openedition.org/norois/ 4729 ; DOI : https://doi.org/10.4000/norois.4729 


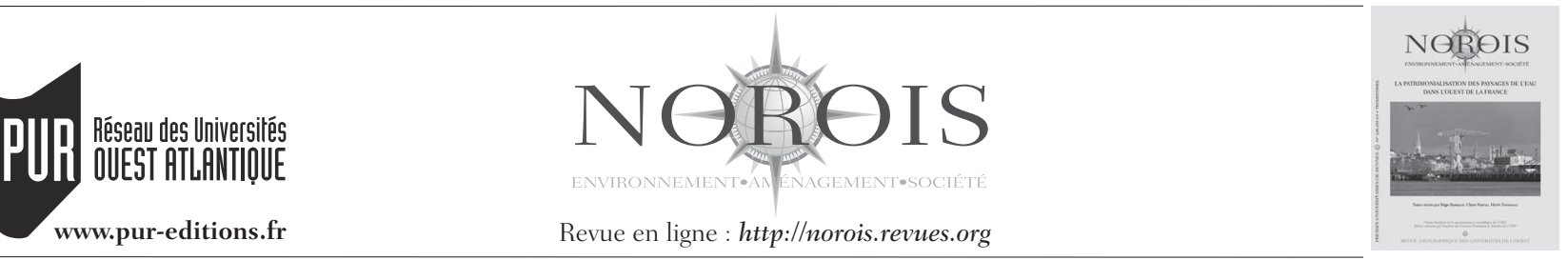

\title{
Les épis de la Loire armoricaine, un héritage à la patrimonialité incertaine
}

\author{
Groynes of the Armorican Lower Loire River, an Heritage with Uncertain Future
}

\author{
Régis Barraud ${ }^{*}$, Nathalie Carcaud ${ }^{\mathrm{b}}$, Hervé Davodeau ${ }^{\mathrm{c}}$, \\ David Montembault ${ }^{\mathrm{d}}$, Claire Pordoy ${ }^{\mathrm{e}}$
}

\footnotetext{
*Auteur correspondant

a Laboratoire RURALITES, Maison des Sciences de l'Homme et de la Société - bâtiment A5, 5, rue Théodore-

Lefèbvre-86000 Poitiens (regis.barraud@univ-poitiers.fr)

b,c,d,e AgroCampus Ouest, centre d'Angers, UMR ESO 6590 CNRS (nathalie.carcaud@agrocampus-ouest.fr), herve.

davodeau@agrocampus-ouest.fr),(david.montembault@agrocampus-ouest.fr),(claire.pordoy@agrocampus-ouest.fr)
}

Résumé : La Loire armoricaine (entre Les Ponts-de-Cé et Nantes) est comprise entre deux pôles de patrimonialisation intense : l'estuaire à l'aval et le Val de Loire inscrit au patrimoine mondial de l'Unesco. Cette section du fleuve est profondément marquée par la crise morpho-sédimentaire et écologique liée à l'abaissement de la ligne d'eau à l'étiage. Cette crise révèle le forçage de la dynamique fluviale du fleuve, dont l'incision du lit engagée dès la fin du XIX siècle, et a été amplifiée par l'extraction sablière et le développement d'un projet d'amélioration de la navigation. Ce projet de la Loire navigable a laissé un héritage imposant constitué d'environ 700 épis qui influencent la physionomie des paysages fluviaux. Aujourd'hui, ces objets pour partie requalifiés par des usages locaux mais pas véritablement patrimonialisés, sont appréhendés en tant que contrainte au rétablissement du bon état écologique et paysager du fleuve. Un projet expérimental de remodelage de ces ouvrages sur une section d’une dizaine de kilomètres a été mené en 2009. Cette opération de restauration écologique a constitué une bonne opportunité pour comprendre le statut des épis dans les représentations sociales des paysages.

Abstract : The present article deals with river groynes on the Armorican Lower Loire River. These small groynes were built at the very beginning of the $20^{\text {th }}$ century to allow large vessels to travel between Angers, (upstream) and Nantes (downstream). Despite considerable investment, transport on the river Loire progressively declined throughout the $20^{\text {th }}$ century and is now practically non-existent. The groynes are, however, still highly visible during low flow periods, as the more so because the river bed is now, on average, 2 meters lower than it was a century ago. Along with industrial sand mining in the river (today banned) and port development in the Loire estuary, the groynes are one of the main causes of the lowering of the river bed, which poses a serious ecological threat. In 2009, experimental reshaping of groynes was carried out in 2010 between the towns of Bouchemaine and Chalonnes-sur-Loire (over 15 kilometers). Socio-geographic surveys and reconstitution of this complex fluvial landscape trajectory aimed at understanding the lack of heritage value of fluvial groynes.

Mots clés : héritage - aménagement fluvial - représentations sociales - politiques publiques - Loire armoricaine

Keywords : heritage - fluvial groynes - social representations - public policies - Loire River 


\section{INTRODUCTION}

En 2009, durant la période d'étiage, des travaux lourds ont été engagés dans le lit mineur de la Loire entre Bouchemaine et Chalonnes-sur-Loire (Maineet-Loire). Les riverains et les derniers touristes de la saison pouvaient alors observer sur le fleuve de mystérieuses pelles-flottantes fouillant le lit sableux du cours d'eau pour y retirer des blocs rocheux. La danse des engins se prolongeait sur les berges avec l'amoncellement des matériaux, progressivement évacués par camion. Quel sens donner à cet étonnant ballet? Il s'agissait d'une opération expérimentale de restauration écologique, destinée à relever la ligne d'eau du fleuve réalisée dans le cadre du Plan Loire Grandeur Nature. Le fleuve « de sable» était en effet "opéré », ingénieurs et techniciens s'affairaient sur les étroites sutures transversales héritées de travaux et de rêves d'un passé encore assez proche, ceux de la Loire navigable. La centaine d'épis raccourcis, arasés et quelques fois supprimés en 2009, sont le produit d'un aménagement de grande envergure entrepris au début du $\mathrm{Xx}^{\mathrm{e}}$ siècle afin d'améliorer les conditions de navigation entre Nantes et Angers, puis de développer un axe fluvial de dimension européenne. Les plus fervents partisans « loiristes » imaginaient alors la Loire comme le trait d'union entre le grand commerce transatlantique international et le marché européen, et en particulier son cœur industriel. Les épis constituent un héritage matériel de ce projet utopiste de la Loire navigable. Maintenus par des programmes de réaménagement et d'entretien coûteux tout au long du siècle précédent ils sont désormais appréhendés en tant qu' « héritage-contrainte », contribuant à la crise hydromorphologique et écologique de cette section du fleuve (figure 1). En effet, depuis un peu plus de cent ans, le lit de la Loire s'est incisé suite à son corsetage progressif (Charrier, 1997 et 2000). Plusieurs facteurs ont participé à contraindre et modifier la dynamique fluviale. Il s'agit de la mise en place des levées insubmersibles, de la valorisation agricole des marges de la bande active, de l'aménagement du bassin à marée et de la chenalisation de l'estuaire. En amont de Nantes, l'installation des épis au début du $\mathrm{Xx}^{\mathrm{e}}$ siècle, puis les opérations de déroctage et l'extraction industrielle du sable après la seconde guerre mondiale ont joué un rôle majeur dans le processus d'incision et de déconnection des annexes hydrauliques. Cette déstabilisation du fonctionnement physique du fleuve a entraîné des changements paysagers et écologiques importants, participant à la dévalorisation de cette Loire « industrieuse », isolée entre deux pôles patrimoniaux : l'estuaire et la Loire des châteaux classée au patrimoine mondial de l'Unesco en 2000. Alors que l'avenir des épis dans le paysage ligérien est mis en question, cet article propose une analyse de la trajectoire paysagère de ces objets et de la Loire armoricaine dont le statut patrimonial semble incertain. Le remodelage des épis a suscité un débat au sein des conseils municipaux mais l'opération n'a pas généré de mobilisation sociale forte pour la défense de ces ouvrages. Est-ce à dire que ces héritages ne font pas partie des éléments qui alimentent la patrimonialisation du fleuve (ports, quais, cales, embarcations, vestiges archéologiques, voire levées)? Le premier temps du développement est consacré à la présentation du terrain d'étude, la Loire armoricaine. Ce prolongement introductif vise à consolider la mise en contexte du développement analytique qui suivra. La deuxième partie porte sur la reconstitution géohistorique de la trajectoire paysagère des épis. Enfin, la troisième interroge les représentations sociales des épis et des paysages associés.

\section{LA LOIRE ARMORICAINE : DU FLEUVE PITTORESQUE AU FLEUVE EN CRISE}

\section{Attractivité et singularité de la Loire armoricaine}

L'analyse des Guides bleus et des Guides Joanne de Sophie Bonin (2001) suggère que la Loire armoricaine constitue, depuis les milieux du XIX ${ }^{\mathrm{e}}$ siècle, l'un des itinéraires les plus attractifs pour le voyageur en quête de sites pittoresques. Mais cette recherche permet aussi de mettre au jour les oscillations du niveau de reconnaissance du fleuve, entre Angers et Nantes. Ainsi, les guides récents semblent démentir l'effet de la crise morpho-sédimentaire sur l'intérêt paysager du fleuve. Le nombre de mentions de «sites d'intérêts » relevés par S. Bonin (2001) dans les publications touristiques a augmenté depuis les premiers guides Joanne jusqu'aux Guides bleus récents, on relève seulement une période de relatif désintérêt dans les années 1950. La Loire armoricaine «d'avant les épis » a été intégrée au réseau 


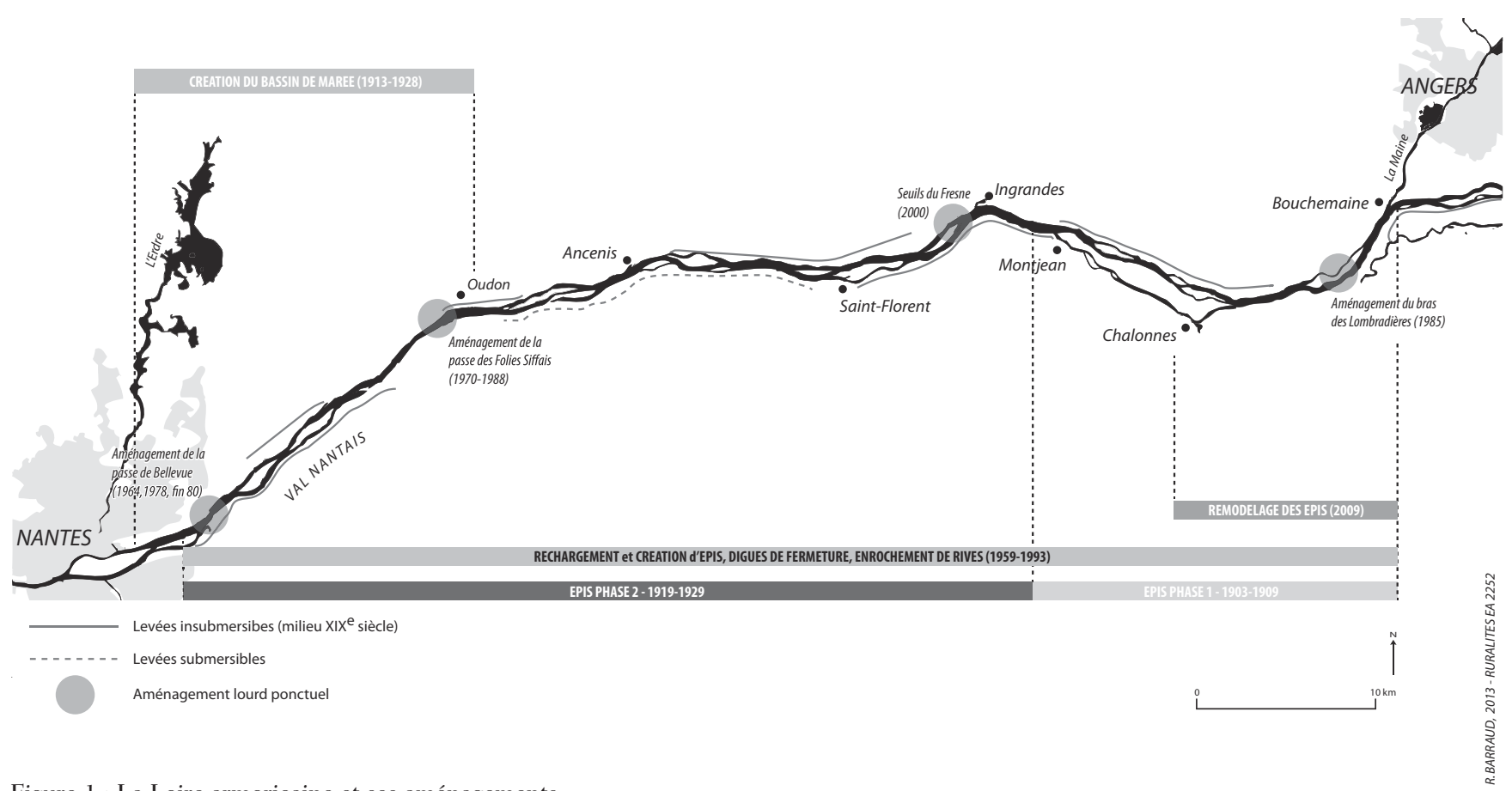

Figure 1 : La Loire armoricaine et ses aménagements. The Armorican Lower Loire River and its hydraulic structures

proto-touristique régional et national par le biais, entre autres, des voyages en vapeur (Chapplain, 1830 ; Barron, 1888). Georges Touchard-Lafosse (1851) souligne l'intérêt des paysages de la vallée dans la traversée des cantons de Chalonnes et de Saint-Georges. Il ressort de ses descriptions la valorisation de la richesse des localités riveraines du fleuve. Il s'agit de la fortune des notables angevins qui s'affiche le long des rives de la Maine et jusqu’à la Pointe, mais aussi de celle issue de la vitalité des systèmes productifs, qu'ils soient agricoles ou industriels. Ainsi, au sujet du canton de Saint-Georges, le journaliste évoque une " "terre promise" [...] riche de produits excellents et variés [...] véritable Eden pour les jouissance de la vue». Il décrit un paysage qui avant lui a été interprété par la sensibilité des artistes et des poètes : "Que d'artistes, assis sur les belles pelouses de la rive droite, ont copié ces délicieux aspects que l'œil saisi à travers les grands léards ou peupliers noirs, qui se balancent dans les îles et semblent darder leur cime verdoyante au fond des eaux du fleuve. Que de poètes admirant cette nature majestueuse ont laissé ici caresser leur imagination au doux bruissement de la feuillée!» Le chroniqueur se laisse impressionner par l'aspect sauvage des coteaux et de leurs « rochers », il valo- rise également la Loire « industrieuse » et ses usines « dont la fumée tourbillonne sur le ciel bleu », sa navigation intense, le trafic des chaufourniers et des bouilleurs de cru. Au cours du siècle suivant, les écrivains tels que Julien Gracq ou encore Maurice Genevoix et Hervé Bazin offrent un éclairage plus précis et subtil sur les paysages de Loire et les relations au fleuve. La poésie de l'écriture de Gracq mêle à la fois l'art de l'évocation (les paysages nous transportent ailleurs par le biais de la mémoire et de l'art) et la sensibilité de celui qui habite les lieux, en particulier lors de l'enfance : «Quand je passe dans les rues de Saint-Florent, ou quand je me promène sur les bords de la Loire, je m'étonne de l'absence de jeux et de cris d'enfants là où, à huit, à dix ans, nos bandes menaient leur train sur le quai et le long des buissons de la rive. À cette époque, on construisait les épis noyés de la Loire navigable : de hautes piles de claies de châtaignier s'entassaient sur la cale : le grand jeu était d'y grimper et de sauter sur le pavé du quai de la plus grande hauteur possible » (Gracq, Lettrines 2, 1974).

Les artistes comme les géographes s'accordent pour attribuer une singularité à la Loire entre Nantes et Angers (Dion, 1961; Coyaud, 2003; Barraud et al., 2010). Ces caractéristiques qui permettent de 
la distinguer nettement de la Loire saumuroise et de l'estuaire sont d'ordre morphologique et culturel. L'Atlas des paysages du Maine-et-Loire (2005) a délimité une unité paysagère spécifique dénommée « La Loire des promontoires ». La limite amont coïncide, à l'aval immédiat des Ponts-de-Cé, avec le dernier grand bec, formé par la confluence de la Maine et de la Loire. En quittant le bassin parisien, la vallée s'encaisse plus nettement et se resserre en devenant plus lisible à l'échelle du grand paysage. Les accidents topographiques captent le regard à l'image de la corniche angevine en rive gauche. Le cours d'eau à fond mobile présente deux formes associées, les îles ${ }^{1}$ et les bras multiples. Les îles occupent une position latérale et dispersent les écoulements dans les bras ${ }^{2}$ où se succèdent les mouilles et les seuils sableux. Le lit majeur est intensivement exploité par l'agriculture (prairies, culture du lin et du chanvre). Cette limite amont est aussi de nature historique, elle épouse les frontières des territoires de l'Ancien Régime et celles de la Vendée militaire. À l'aval, le système estuarien impose une limite mobile qui remonte aujourd'hui au-delà d'Ancenis. Cependant, l'entrée dans la ville de Nantes constitue toujours une limite plus franche.

\section{De la perception de la crise aux projets de valorisation patrimoniale}

La crise écologique et paysagère est perçue par les acteurs locaux depuis la fin des années 1970 et la sonnette d'alarme est tirée par les élus au début de la décennie suivante : «Les îles ont soif », «du sable à perte de vue au mois de juin, on a jamais vu ça! » (Propos du Maire d'Ancenis, E. Landrain, 1981). L'arrêt des extractions entre Nantes et Oudon est acté dès 1977 et l'interdiction est totale en 1995. La déconnection du cours de la Loire et de ses affluents entraîne la construction d'ouvrages hydrauliques tel que le barrage mobile de Pont-Rousseau sur la Sèvre nantaise (1995). Un premier programme de remontée de la ligne d'eau est défendu par l'Établissement Public d'Aménagement de la Loire et de ses Affluents (EPALA) en 1990. Cet objectif prioritaire est intégré au premier Plan Loire (1994), mais

\footnotetext{
1. La dynamique fluviale forcée par les aménagements a conduit à une réduction drastique du nombre de ces îles depuis le XVII ${ }^{\mathrm{e}}$ siècle. Ainsi, on dénombrait 67 îles entre Ingrandes et Nantes en 1665 (carte de Grion), il en subsiste aujourd'hui seulement 19 .

2. Annexes hydrauliques et boires.
}

les actions engagées sur la section armoricaine ne sont pas à la hauteur du problème (Zeimert, 1998, Sauvegarde de la Loire angevine). La charte (2007) du Comité Loire de Demain (CLD) qui fédère les riverains et les associations locales, exprime plus précisément les causes et les effets de la crise morpho-sédimentaire : «La Loire armoricaine subit un sinistre environnemental depuis plus de trente ans : chute de la ligne d'eau d'étiage, assèchement de la vallée, accélération du courant, érosions, amplification de la marée, de l'envasement, de la salinité... Les conséquences sont lourdes, portant atteinte à l'ensemble du patrimoine fluvial et aux différents usages : paysage, ressource en eau, biodiversité, cadre de vie, pêche, nautisme, attrait touristique. En cause, les aménagements pour la navigation fluviale et maritime conjugués aux dragages et à la suppression des seuils. »

La patrimonialisation du grand Val de Loire institutionnalisée par le classement UNESCO en 2000 au titre des paysages culturels entre Sully et Chalonnes-sur-Loire recoupe en partie le cours de la Loire intégré au Parc naturel régional LoireAnjou-Touraine en 1996. Parallèlement à la reconnaissance d'un «fleuve à visage humain ${ }^{3}$ ", le Plan Loire Grandeur Nature concrétise à l'échelle de l'ensemble du bassin une démarche de restauration de la qualité écologique du cours d'eau depuis 1994 (Baron-Yelles, 2006; Rode, 2010). Notons qu'en 1990, un projet porté par l'EPALA visait à articuler autour des cours principaux de l'Allier et de la Loire, un dispositif de double préservation/valorisation du patrimoine associant un parc national (lit mineur) et un Parc naturel régional (PNR). À l'aval, la patrimonialisation de l'estuaire est éclatée entre plusieurs acteurs mais elle est très active, et se renforce sans cesse depuis le milieu des années $1990^{4}$. Entre ces deux pôles de valorisation qui ont aspiré d'importants investissements publics, la Loire armoricaine fait figure, pour les acteurs locaux, de parent pauvre. Les actions entreprises depuis 1992 en matière de connaissance, de sensibilisation et de préservation des paysages par le Conservatoire des Rives de la

\footnotetext{
3. Expression employée dans les documents de communication du site Unesco : [http://www valdeloire.org/Patrimoines/Patrimoine-mondial/Criteres-de-l-inscription-UNESCO].

4. Création de l'association Estuarium en 1994; la Cellule de Mesures et de Bilan en 1998 devient GIP Loire Estuaire en 2004; une Maison de l'Estuaire sera créée à Saint-Nazaire en 2015 à l'initiative de la Région, de la ville de Saint-Nazaire et du Grand Port Maritime. Le patrimoine industriel estuarien fait aussi l'objet de multiples initiatives de valorisation.
} 
Loire et ses affluents (de Montsoreau à l'Océan), ont visé à atténuer les effets de la crise mais cette structure n'a pas vocation à résoudre le problème d'abaissement de la ligne d'eau.

Cette Loire armoricaine en crise est pourtant riche de très nombreux héritages. Au-delà des objets classiques bien documentés (ports fluviaux, fours à chaux, fermes chanvrières, châteaux médiévaux), l'extraction de sable et l'incision du lit ont mis au jour un grand nombre d'éléments expertisés par l'archéologie fluviale (Ménanteau et Poissonnier, 2002). Il s'agit d'aménagements médiévaux à l'exemple des épis de pêcherie du Grand Aireau, de chaussées de moulins mais également de pirogues de l'âge de Bronze, de chalands et autres bois d'épaves. Les fouilles en lit majeur révèlent aussi d'importantes ressources pour la compréhension des interactions nature-sociétés sur le temps long. Un mouvement associatif diffus participe à la valorisation de cet héritage. Des initiatives de mise en cohérence ont été tentées depuis le début des années 1980, notamment par l'action de P. Cayla, fondateur de l'écomusée de Montjean-sur-Loire (1981-2003) puis de l'association «Patrimoine culturel Loire » dont l'objectif initial était la définition d'un « projet fédératif ou confédératif de recomposition des structures de valorisation du patrimoine fluvial ligérien » (Cayla, 2007). Les épis, hormis les ouvrages médiévaux exhumés par l'industrie sablière et l'érosion, font rarement l'objet d'un discours patrimonial ou d'initiative de sauvegarde. On relève toutefois des références esthétisantes dans les beaux livres consacrés à la Loire. Cette esthétisation est parfois reprise dans la rhétorique touristique à l'exemple de l'île de Béhuard ${ }^{5}$ ou dans le diagnostic paysagiste (dossier d'enquête publique préalable au remodelage des épis, 2009). Pour comprendre le statut actuel de ces ouvrages dans le paysage de la Loire armoricaine, il convient de revenir sur les raisons initiales de l'aménagement, les réactions des riverains et les conditions du maintien de ces aménagements jusqu’à aujourd'hui.

5. «Engloutis l'hiver, ils apparaissent l'été. Ce sont des points forts du paysage qui rythment les grandes grèves de sable. Les épis et les bancs de sable marquent, en période d'étiage, l'horizontalité du fleuve et apportent de riches variations dans les couleurs amenant harmonie entre la nature ligérienne, sable, ciel et Loire ». Extrait de la plaquette produite par Angers Loire Tourisme en 2011 : [http://www.angersloiretourisme.com/ Upload/communes/BEHUARD_2011.pdf]

\section{AménAgement des ÉPIS, MOBILISATIONS ET REPRÉSENTATIONS SOCIALES : SYNTHÈSE GÉOHISTO- RIQUE (DU DÉBUT DU XX $\mathbf{X}^{\mathrm{E}}$ SIÈCLE À AUJOURD'HUI)}

\section{Origine et contexte d'émergence du projet d'aménagement de la Loire navigable}

Le projet de la Loire navigable repose à la fois sur le souvenir nostalgique d'un relatif âge d'or de la batellerie en Loire au XIX ${ }^{\mathrm{e}}$ siècle et sur une utopie : créer un axe de communication puissant reliant la façade atlantique à Bâle et à l'Europe centrale. La Loire est même perçue comme un trait d'union potentiel entre ce cœur industriel européen et l'Amérique du Nord (Zimmermann, 1905). Ce projet serait assuré par l'amélioration des conditions de navigation de la Loire entre les ports de Nantes/ Saint-Nazaire et le canal de Briare. L'objectif du projet relève d'enjeux économiques qui ont d'abord trait au développement du port de Nantes (d'Andigné, 1928). La dispute sur le choix technique à mettre en œuvre opposait les partisans du canal à ceux privilégiant l'aménagement à courant libre (VauthierVézier, 2007). À cette époque, une conjonction de facteurs avait conduit au déclin de la navigation de l'axe Nantes-Orléans : l'évolution du régime du fleuve (Charrier, 2000) et bien sûr l'arrivée du train au milieu du XIX ${ }^{\mathrm{e}}$ siècle. En 1855, il existe encore 4 lignes régulières entre Nantes et Orléans équivalent à un trafic d'environ 10000 bateaux par an. L'imagerie des vapeurs et de leur bonne société profitant du charme des voyages pittoresques sur le fleuve semble gommer les extraordinaires difficultés à assurer l'entretien d'un chenal mobile : cette image glorieuse va longtemps perdurer.

Le projet d'amélioration de la navigation n'est pas porté par l'administration mais par un réseau de lobbying local qui se structure en 1893. Ainsi, est créée la Société d'initiative et de propagande pour l'exécution d'une voie navigable entre Nantes et Orléans et «prolongements », rapidement rebaptisée « Loire navigable ». Elle est fondée à l'initiative du sénateur de Loire-Inférieur (Charles Lecour-Grandmaison), du maire de Chalonnes (Lucien Frémy) et de divers acteurs du monde économique des deux départements. Dans la foulée, en 1894, le conseil général 
de Loire-Inférieure émet auprès du gouvernement la demande de réalisation d'une voie navigable entre Nantes et Orléans «tout en respectant les droits acquis ». L'association déploie une activité de propagande remarquable au début du $\mathrm{xx}^{\mathrm{e}}$ siècle (comités locaux, pétition, journal, congrès).

De premiers travaux d'essai sont tentés dès 1903 vers Montjean mais les résultats obtenus sont désastreux, les ouvrages légers inadaptés sont emportés par le courant ${ }^{6}$. La réalisation des épis sur la section expérimentale comprise entre Bouchemaine et Chalonnes est déclarée d'utilité publique (figure 2). La section d'essai de 9 kilomètres est rapidement portée à 14 kilomètres avec l'intégration du bras de Chalonnes jusqu'à Montjean, stratégique sur le plan industriel (charbon, chaux). Le coût des travaux est estimé à 1,8 million de francs. Une enveloppe financière globale de 14 millions de francs est prévue pour mener à bien les travaux entre Nantes et la Maine en cas de résultats positifs sur la section d'essai. Sur le plan technique les travaux sont inspirés des travaux récents effectués sur le Rhône (resserrement du lit par des digues et épis), comme l'indique la note de M. Zimmermann publiée dans les Annales de Géographie en 1905. Cet auteur ajoute que l'ingénieur Guénot en charge du projet d'aménagement de la Loire sur la section d'essai était un élève de Girardon, responsable de la conception des travaux de correction du Rhône. La technique des épis noyés semble avoir été adaptée sur ce dernier fleuve à partir d'expériences réalisées en Allemagne (Tricart et Bravard, 1991) ${ }^{7}$. La réduction de la largeur du chenal à 150 mètres emploie des «épis noyés » : «Les ouvrages sont composés d'épis, ou pieux espacés, reliés par des panneaux en perches de châtaigniers et contrebutés, le cas échéant, par des enrochements. Selon les lieux, ils sont eux-mêmes reliés les uns aux autres, à certains points par des digues longitudinales, construites de la même façon » (Schwob, 1900). L'objectif initial est d'obtenir un chenal régulier, auto-entretenu par le courant, d'un tirant d'eau de 1,50 mètre. Les ouvrages légers en clayonnage sont finalement rem-

6. Cet échec rappelle les difficultés rencontrées pour aménager ce fleuve à fond mobile, lors de projets précédents, notamment ceux conduits par l'ingénieur Lemière (1834-1840) qui concernent marginalement la Loire en amont de Nantes (estacades de fermeture de boire, digues au droit du port d'Ancenis).

7. Ces deux auteurs analysaient déjà les effets morphologiques et écologiques des grands aménagements hérités sur le Rhin, le Rhône et le Danube. placés par des ouvrages renforcés (pieux battus et enrochement). Dans les faits, la chenalisation du cours de la Loire armoricaine qui est prolongée jusqu'à Oudon entre 1922 et 1928, mobilise différents ouvrages : (1) l'épi de convexité destiné à fixer les sables, (2) la digue longitudinale destinée à diriger les eaux et obtenir les profondeurs voulues ainsi que la jonction des mouilles, (3) la traverse ou le tenon de renforcement de la digue, (4) le barrage de faux-bras, (5) l'enrochement de berges.

La création du bassin à marée est également déclarée d'utilité publique en 1913, et des travaux conséquents sont réalisés entre 1925 et 1928 afin de permettre la propagation de la marée et l'autocurage du chenal. Progressivement « l'estuarisation » des politiques publiques d'aménagement entre en contradiction avec la fonctionnalité du tronçon fluvial Nantes-Angers. Les travaux effectués et la réponse physique du fleuve disqualifient la batellerie traditionnelle.

\section{Des travaux contestés : création de l'association de défense des riverains de la Loire}

Les travaux d'essai (La Possonnière, Montjean) sont achevés en 1908, ils donnent lieu à de très vives protestations : accélération des courants, érosion de terres agricoles, modification de l'hydraulique des bras secondaires, relèvement du plan d'eau. Les usages tels que le rouissage du chanvre sont sévèrement perturbés. À partir des années 1910 la contestation prend de l'ampleur alors que le fleuve est marqué par une succession d'années à forte hydraulicité et par des crues importantes, dont bien sûr la grande crue de $1910^{8}$. Mais la vulnérabilité accrue des paysans est liée principalement à l'exploitation agricole de parcelles basses conquises sur la bande active (Charrier, 2000). L'association de défense des riverains est créée en 1910. Elle rassemble très rapidement de nombreux riverains de la Loire armoricaine, et compte près de 600 adhérents au début des années 1920. Elle est composée d'agriculteurs qui font tous état des mêmes difficultés à exploiter les parcelles les plus basses

8. Selon des ingénieurs de l'époque : les riverains ont supporté 19 crues supérieures à $3,5 \mathrm{~m}$ en 4 ans et 6 mois, alors que de 1903 à 1908, soit en 5 ans, il ne s'en était produit que 7 (rapport de l'ingénieur en chef des Ponts et Chaussées, 1913 - 3 S 384, ADLA). 
(cultures, pacage et foins) et à utiliser les annexes hydrauliques. L'ensemble des communes riveraines adhère à l'association et les membres les plus virulents dans l'affrontement avec l'administration des Ponts et Chaussées sont des élus locaux qui occupent parfois des positions importantes au plan local (maires, présidents de syndicat de marais, députés, sénateurs). Parmi eux on retrouve des «maîtres de la terre » très conservateurs et pour la plupart antirépublicains. L'association est aussi fortement soutenue par le syndicat agricole de l'Anjou et l'Union des Syndicats Agricoles de l'Ouest. L'institution départementale a contesté dès le départ les travaux projetés et a refusé d'y contribuer financièrement. L'amélioration de la navigabilité du fleuve est clairement défendue par la Chambre de Commerce et d'Industrie du Maine-et-Loire qui y voit un moyen de dynamiser le port d'Angers.

\section{Les moyens d'actions de l'association : concertation, contre-expertise et recours juridique}

Les riverains utilisent des moyens très modernes, comparables en certains points avec les méthodes et stratégies utilisées aujourd'hui par les associations de protection de l'environnement. Le premier moyen mis en oeuvre est celui de la concertation, à l'exemple de l'enquête collective effectuée sur le terrain en 1922. Cette visite réunit, à la demande de l'association, une quarantaine d'acteurs locaux et les agents administratifs en charge de l'exécution des travaux. L'association n'hésite pas à faire réaliser une contre-expertise, comme c'est le cas au début des années 1920. L'association de défense des riverains utilise prioritairement l'assemblée départementale pour donner une portée politique à ses requêtes. En 1921, elle fait adopter au Conseil Général une résolution qui demande :

«1- Que tous les travaux soient arrêtés;

2- Que les épis, barrages, enrochements, y compris les sables accumulés soient enlevés dans le plus bref délai possible, afin d'assurer la sauvegarde des terrains, la sécurité des levées, l'existence des habitants des vallées et de leurs propriétés par le libre écoulement des eaux.

3- Que le Parlement vote un crédit afin d'indemniser équitablement les riverains en proportion des

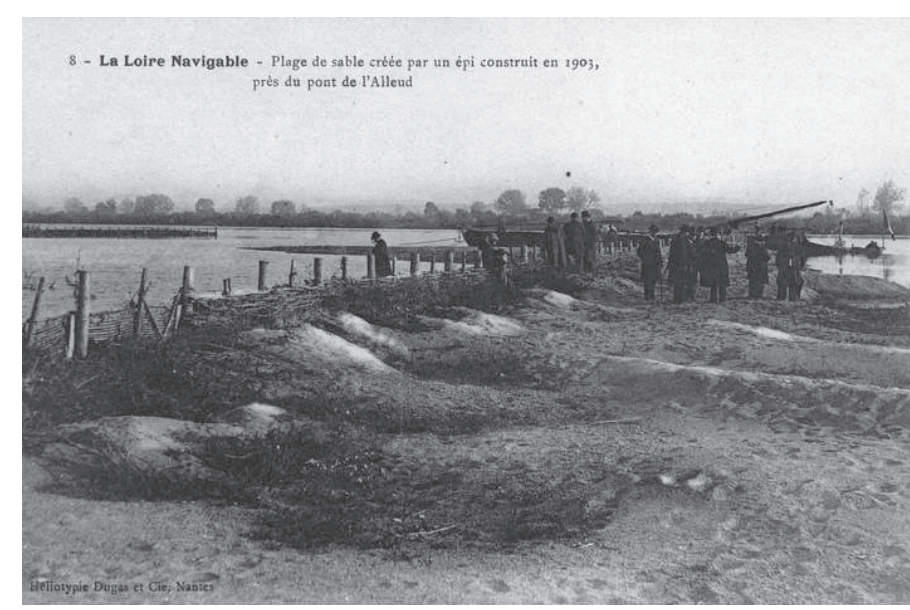

Figure 2 : Le chantier d'installation des épis (phase 1, cf. figure 1) Bouchemaine

Old postcard showing the construction of the groynes in 1903 in

Ouvrage en clayonnage construit à Bouchemaine en 1903. Carte postale ancienne. Issue d'une galerie constituée par l'association d'Histoire des Coteaux de Loire et de Maine.

pertes subies par eux depuis 16 ans et de la dépréciation de leur propriétés. »

Face à ces plaintes, l'administration est assez intransigeante : dans un premier temps elle réfute systématiquement l'incidence négative des travaux entrepris sur l'activité agricole. Selon les ingénieurs, les dégâts seraient davantage liés à la succession d'années à fortes crues. Les Ponts et Chaussées finissent par reconnaître les effets des épis et chevrettes barrant les bras secondaires mais uniquement dans des cas localisés (bras de Chalonnes par exemple). Suite à l'enquête de 1922, l'association a regroupé les plaintes de nombreux riverains. En 1928, plus de 541 demandes d'indemnités ont été recensées et transmises à l'état, cela représentant une superficie de 1800 hectares. La tension est vive entre l'administration et les opposants jusqu'à la fin des années 1920. Certains opposants proposent même la suppression des épis, une partie d'entre eux seront effectivement arasés vers 1929.

Pourtant, dans le même temps, une approche plus sensible du paysage de la Loire aménagée semble émerger. Ainsi, la Loire des épis apparaît dans les écrits de Julien Gracq comme un nouveau terrain de jeux où se forgeront des générations de souvenirs d'enfance. La presse laisse également place à des interprétations esthétisantes comme lors de l'été 1937. Un chroniqueur y compare les paysages de la Loire à la Pointe (Bouchemaine, Maine-et-Loire) 
à ceux des marais salants de Guérande. L'horrible vision des épis "marque de la torture infligée à la Loire » (d'Andigné, 1928), semble cohabiter avec l'approche utilitaire des usagers de la Loire qui perçoivent ces ouvrages comme des obstacles à l'écoulement, comme des contraintes techniques à supprimer mais également avec des regards bienveillants, ceux de l'enfant ou du promeneur. Les épis peuvent aussi être admirés en tant qu'œuvre de l'ingénieur par les partisans du projet d'aménagement.

\section{Le rebond de la mobilisation sociale : le tournant environnemental (fin des années 1980)}

Après plusieurs décennies d'entretien du chenal navigable par rechargement des épis, les effets combinés de l'exploitation industrielle et de la chenalisation commencent à susciter des inquiétudes locales. En 1985, l'ouverture à la navigation du bras des Lombardières suscite des tensions entre l'administration et les riverains. En 1989, un ultime projet de mise en valeur de la Loire fondé sur le développement de la navigation de tourisme à l'amont d'Angers constitue un élément clé du renouvellement de la mobilisation des riverains de la Loire. Ce projet consistait à prolonger l'aménagement des épis à l'amont d'Angers. L'Association de sauvegarde de la Loire Angevine est créée en 1989, elle intégrera des collectifs militants structurés à l'échelle régionale (La Sauvegarde de l'Anjou) et à l'échelle nationale (Loire vivante). Les associations environnementales dénoncent l'impact de l'exploitation sablière industrielle et des travaux d'entretien et d'amélioration du chenal. L'arasement de seuils rocheux naturels sur le site de Bellevue au milieu des années 1970 est particulièrement critiqué et identifié comme un facteur de dégradation du fleuve.

La mise en place du plan Loire Grandeur Nature (1994) et le déclin de la navigation concrétisent le tournant environnemental qui caractérise l'évolution des mobilisations sociales et des politiques publiques à l'échelle du bassin de la Loire. Cependant, les associations locales qui militent pour la restauration écologique et paysagère de la Loire entre Angers et Nantes sont insatisfaites du niveau d'intégration de cette section du fleuve dans la mise en œuvre des politiques publiques. Afin de faire pression sur les pouvoirs publics et d'influer sur les politiques publiques, la mobilisation associative se renforce encore en 2005 avec la création du Comité Loire de Demain ${ }^{9}$. Ce collectif regroupe 350 particuliers, 33 associations et 24 communes riveraines. Cette évolution du mouvement local intervient juste avant le terme du deuxième Plan Loire (2000-2006) et après l'aménagement expérimental très discuté des seuils artificiels du Fresne-sur-Loire destinés à relever la ligne d'eau d'étiage (2004).

L'association se distingue par sa volonté à produire des contre-expertises et de proposer des solutions d'aménagement alternatives et complémentaires à celles mises en œuvre jusqu'à présent. D'une manière générale, Loire de Demain juge l'action publique insuffisante pour infléchir de manière significative l'évolution du fleuve. Notons également que le renouvellement de la mobilisation des riverains de la Loire armoricaine s'inscrit dans la filiation des combats des années 1910-1930 (association de défense des riverains). Cette histoire militante est connue par les dirigeants du collectif et elle est parfois mobilisée dans les discours (Zeimert, 1998). Ainsi, il existe une défiance historique entre les populations locales et l'administration en charge de l'aménagement et de la gestion du fleuve. De nouveau, les militants font valoir, face à l'expertise conventionnelle, la pertinence des savoirs locaux et des compétences d'usagers, de riverains du fleuve. Plusieurs cadres du collectif ont effectué des formations scientifiques universitaires (géographie, écologie, histoire). Cette mobilisation sociale témoigne d'un tournant environnemental dont il faut évaluer les effets sur les perceptions sociales du fleuve et de ses abords : comment les épis sont-ils vécus et perçus au moment où le programme expérimental de remodelage est engagé (figure 3) ? Une valeur patrimoniale leur est-elle attribuée? Si oui, laquelle et par qui?

\footnotetext{
9. Le CLD a été créé à « l'initiative d'Yves Borbeau, membre fondateur de l'Association Nationale des Plaisanciers en Eau Intérieure » : [http://www. loire-de-demain.fr/Dossiers/2013-03-23-AG-presentation.swf].
} 


\section{LES REPRÉSENTATIONS SOCIALES DES ÉPIS ET DES PAYSAGES DE LA LOIRE ARMORICAINE}

\section{Méthodologie : couplage d'une approche quantitative exploratoire et d'une analyse qualitative}

En complément d'une approche bibliographique géohistorique pour comprendre l'histoire des épis de Loire, plusieurs outils sont mobilisés : le dépouillement de l'enquête publique, l'analyse des discours associatifs, le recueil de photographies issues d'un concours du journal Ouest-France sur "vos plus belles photos de Loire » (390 clichés), l'analyse de dessins d'enfants (projet « Rivière d'images et Fleuve de mots »), la mise en place d'une enquête en ligne auprès d'étudiants et d'associations environnementales (350 personnes) et d'entretiens auprès de riverains et d'acteurs clés du projet de remodelage $(20)^{10}$. Ces outils visent les mêmes objectifs : amener les personnes interrogées à décrire leurs pratiques dans la vallée, les faire s'exprimer sur le paysage de Loire et ses dynamiques, pour progressivement aborder les épis (comment nommer cet objet ${ }^{11}$, à quoi sert-il, etc.) puis les travaux de remodelage (quel est l'objectif?). Les deux outils sont construits avec un support photographique, mis en œuvre selon des dispositifs inspirés par les travaux d'Y. Michelin (1998) et d'E. Bigando (2008), pour tenter de saisir la place des épis dans les représentations paysagères. Vingt images ont été soumises aux personnes interrogées ( «laquelle est la plus/moins représentative des paysages de la vallée de la Loire ») en fonction de critères couplés selon la prise de vue (détail ou panoramique, été ou hiver, vue du ciel ou du sol), les éléments structurants (Loire armoricaine ou non, présence ou absence : des épis, du fleuve, du sable, du bâti, de l'agriculture, de la navigation).

10. Les enquêtes ont été menées par Appoline Taillade (Master 1, université d'Angers, 2010) et par Claire Pordoy (M2, histoire de l'Art, Rennes, 2011 ), stagiaires à l'AgroCampus Ouest d'Angers dans le cadre du programme PATRA - l'encadrement des stagiaires a été assuré par H. Davodeau et le traitement des résultats des enquêtes a été conduit par D. Montembault

11. Les habitants ne nomment pas toujours les épis ainsi : ils utilisent les termes de «barrages » pour leur forme perpendiculaire aux rives et le léger ralentissement du courant en amont de l'épi (qui «barre » le passage) et entraîne un affleurement de l'eau sur la partie sommitale de l'épi; mais aussi "épis noyés » car à leur construction, la partie sommitale était en effet placée à la côte 0 , ils étaient ainsi invisibles même pendant la période des basses eaux; enfin « digues » au regard de la morphologie des ouvrages.

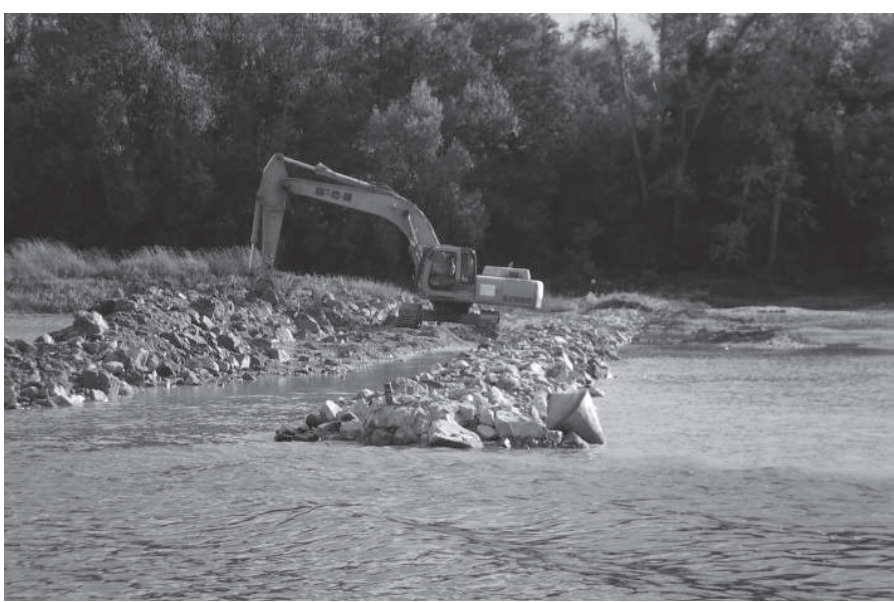

Figure 3 : Le chantier de remodelage des épis (été 2009) Restructuration of the groynes in 2009

Les travaux de remodelage (79 épis) consistent à abaisser $(50 \mathrm{~cm}$ en moyenne) et/ou à raccourcir les épis de manière à élargir le chenal au maximum de 50 mètres. Les travaux ont été accompagnés par des actions d'arrachage de la Jussie et par la dévégétalisation. Le coût des travaux s'élève à 3,2 millions d'euros (Région, Agence de l'Eau, Établissement Public Loire, État, Feder). La maîtrise d'œuvre est assurée par Voies Navigables de France. L'objectif est notamment de relever la ligne d'eau à l'étiage de 25 à 35 centimètres. Un suivi de cinq ans est engagé pour évaluer les effets et décider du prolongement de l'opération vers l'aval. Pour plus de précision sur ce plan : [http://www.loire-estuaire.org/comite_estuaire/reunions.html].

\section{L’analyse des usages : les épis intégrés au cadre de vie}

La phase de terrain a permis de construire une première typologie des pratiques sociales sur et autour des épis. Pour les pêcheurs, ils facilitent l'accès au lit mineur et, en tête d'épi coté aval, l'accès à une « fosse » poissonneuse résultant du léger dénivelé entre l'accroche et la tête de l'ouvrage. Par ailleurs, les épis servent de point d'amarre pour les barques. Ces pratiques sont celles d'usagers réguliers de la Loire (souvent depuis l'enfance) et s'intensifient avec la baisse des eaux au printemps. Les baigneurs, quant à eux, profitent de « l'amorti » entre les épis, et du sable accumulé (les grèves). Là encore, ces pratiques sont celles de ligériens de longue date, bons connaisseurs des risques du fleuve (les «cul de grèves ») et des lieux de baignade à l'écart (cette pratique est interdite depuis 1960). Les épis sont aussi des lieux de pique-nique (barbecue) : entre les épis pour profiter du sable et creuser le foyer du feu, et sur pour recueillir le bois mort amassé. Ils sont aussi un évènement de la promenade en bord de Loire (figures 4 et 5). S'aventurer au bout des épis agrémente la marche parallèle au fleuve. L'accès à la 

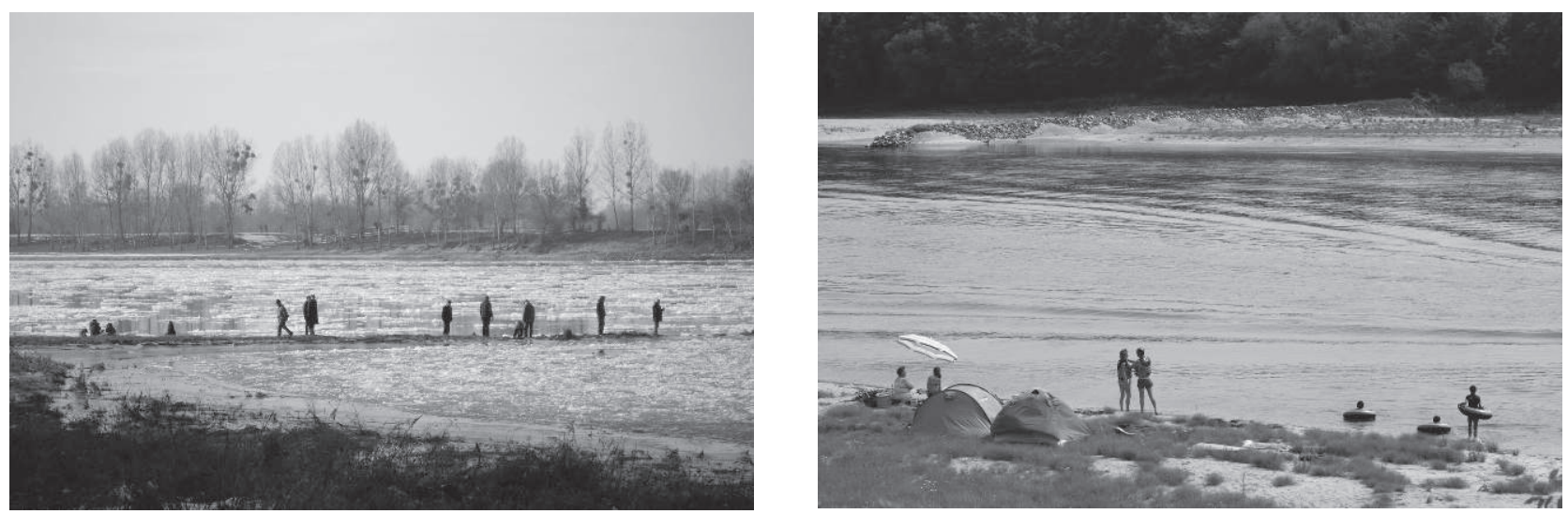

Figures 4 et 5 : Les épis discrètement requalifiés par les usages locaux

Alternative uses of the groynes: Exploration of the frozen river in 2012 od transformation into beaches during the summer Les ouvrages permettent l'exploration du fleuve gelé - cliché N. Carcaud, 2012. Les grèves qui se développent entre les épis se muent en plages durant la période estivale - cliché C. Portal, 2012).

tête de l'épi est une petite aventure (grosses pierres, pieux) récompensée par une immersion dans le lit du fleuve (prise de vues photographiques) avec des sensations plus fortes (la puissance du courant, la «nature ») que celles captées depuis les berges plus fréquentées (un fleuve plus distant et plus nonchalant). Plus anecdotique, les épis sont des réceptacles : la promenade sur les épis est aussi l'occasion de découvrir ce que dépose le fleuve. Les archéologues y font de réelles découvertes qui attisent la curiosité du promeneur du dimanche.

\section{Les modèles de perception du fleuve : des clichés patrimoniaux (Unesco, nature sauvage) aux références vernaculaires}

Ces pratiques sont significatives d'un rapport de proximité étroit entre la population et le fleuve et participent de la définition d'une posture paysagère des gens du lieu (Droz et al., 2009), qui contraste avec d'autres pratiques de loisirs qui se développent autour du fleuve, mais à distance respectable des épis : aménagements des sentiers de "La Loire à vélo », aires de pique-nique, points de vue aménagés depuis les promontoires, sports collectifs sur les grèves (tournoi de volley), motonautisme dans le fleuve. Les premiers résultats des enquêtes ${ }^{12}$ sont très paradoxaux quant à l'attachement des riverains aux épis. Les 261 étudiants et 91 associatifs enquê-

12. Une présentation plus détaillée et illustrée des résultats de cette enquête a été réalisée dans le cadre du rapport final du programme PATRA rédigé par Hervé Davodeau (2013). tés semblent dans un premier temps exprimer une apparente uniformité de points de vue quant aux images choisies comme significatives des paysages de la vallée: des vues du ciel, prises en été, en format panoramique, avec une légère surreprésentation de la Loire non armoricaine (figure 6). À l'inverse, les moins représentatives sont les photos prises au niveau du sol, présentant des vues de détail, en hiver, avec la Loire en crue et une surreprésentation de la Loire armoricaine. La question portant sur les lieux emblématiques de la vallée de la Loire montre également peu de différences entre les étudiants et les associations. Les deux groupes citent d'abord les éléments culturels valorisés par l'UNESCO (et que l'on retrouve plutôt en Loire moyenne). En second lieu, ce sont des éléments locaux qui sont cités comme emblématiques par les deux groupes. Enfin, les composants naturels caractérisent aussi le fleuve, de façon plus marquée pour les associations. Si la levée a été quelquefois citée (8 fois sur 357 personnes), aucun des ouvrages liés à la navigation ne l'a été.

Sur le contenu même des images, la différence entre les deux groupes de photos est peu marquée également. Cependant, parmi les photos les plus représentatives, on note clairement l'absence des activités (navigation, agriculture et bâti invisibles), et à l'inverse leur présence est remarquée dans le groupe de photos jugé le moins représentatif. Il semble donc que les aménagements humains sont exclus du paysage ligérien. Pourtant les épis sont surreprésentés dans les photos jugées les plus repré- 
sentatives et sous-représentés dans les photos les moins représentatives. Cela soulève une première contradiction.

\section{La perception des épis : entre présence et absence}

Les épis sont presque absents des images diffusées par les médias traditionnels (brochures touristiques, ouvrages grand public, cartes postales, etc.), que ce soit à l'époque de leur construction ou aujourd'hui. Ils n'appartiennent pas aux paysages de référence construits au fil du temps, pourtant ils se retrouvent dans le discours des habitants les plus anciens. Ainsi, les épis sont présents sur deux des photos jugées les plus représentatives des paysages de la vallée. De plus, sur les dessins réalisés lors des entretiens, ils apparaissent 5 fois sur les 13 représentations. Pour autant, leur présence ne contredit pas la représentation du «fleuve sauvage ». Pour les personnes qui perçoivent les épis dans les paysages, le remodelage est en général bien accepté car ils considèrent leur fonction initiale obsolète et connaissent les dommages écologiques qu'ils causent. L'intégration du discours savant (écologie) permet de renforcer l'argumentaire des élus locaux sur la dévalorisation de la Loire en tant que ressource territoriale (dégradation des conditions d'accès, de pratique et de la qualité esthétique d'un fleuve "sans eau»).

L'hypothèse que nous formulons à travers ces premiers résultats d'enquête est une opposition entre les épis tels qu'ils sont vus, les épis tels qu'ils sont sus et les épis tels qu'ils sont vécus : "vus », ils sont naturellement intégrés au paysage ligérien, «sus » ils sont associés au modèle écologique dominant et sont donc exclus de la Loire, "vécus » ils sont à nouveau intégrés à la Loire. Par ailleurs, les épis ne semblent pas avoir une position définie entre patrimoines naturel et culturel : ils sont même plus facilement associés à la Loire «sauvage ».

\section{Le remodelage des épis perçu par les usagers : entre intégration du discours écologique et vigilance}

L'opération de remodelage s'inscrit dans la stratégie globale de restauration physique du fleuve, elle a porté sur l'abaissement et/ou le raccourcissement de 79 épis (cf. figure 3). Deux questions interrogent

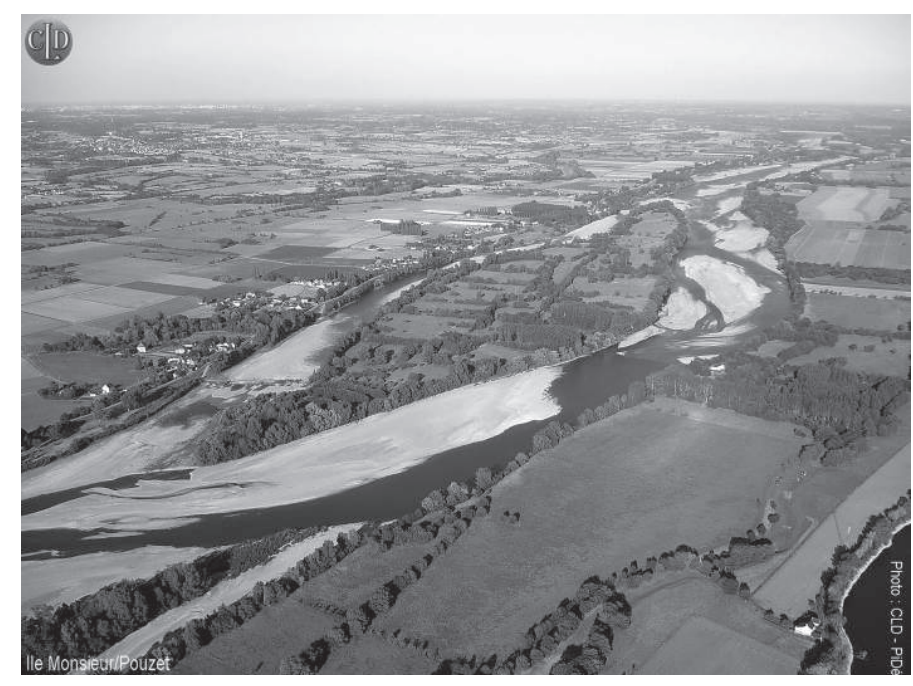

Figures 6 : La Loire armoricaine perçue par les étudiants et les adhérents d'associations environnementales

Common and incommon perceptions of the Armorican Loire River by students and environmental associations members

La figure 6 fait partie des vues les plus représentatives du paysage fluvial ligérien entre Nantes et Angers selon les personnes enquêtées. Il s'agit ici d'une vue aérienne de l'île Monsieur (cliché CLD, mi-juin 2011).

le ressenti de la population dans la trame du questionnaire en ligne adressé aux associations et dans le questionnaire pour les entretiens : «Connaissezvous la raison des travaux? » puis «Qu'en pensezvous? ». Parmi les 96 personnes membres d'associations de protection de l'environnement ayant répondu au questionnaire, $55 \%$ sont au courant du remodelage des épis. Plus des deux-tiers ont proposé une explication aux travaux. D'une manière générale, l'objectif de relèvement de la ligne d'eau est donc connu et associé en premier lieu à la remobilisation du sable accumulé entre les épis vers le fond du lit ainsi qu'à la reconnexion du fleuve avec ses annexes hydrauliques (les boires). L'objectif de biodiversité est plus facilement avancé que celui de la lutte contre le creusement du chenal (qui lui est pourtant associé). Quelques contradictions nous semblent marquantes : la hausse et la baisse du débit sont mises en avant à plusieurs reprises, ce qui est probablement dû à une confusion entre débit et hauteur d'eau. Favoriser la navigation sur le fleuve apparaît plusieurs fois, ainsi que la protection des «zones construites et inondables par un renforcement des levées »! Les réponses, dans leur majorité, valorisent l'objectif d'un retour à un régime fluvial plus naturel, d'un «fleuve plus libre » et moins «corseté » par les aménagements. 
Avant les travaux, l'enquête publique qui s'est tenue entre le 20 avril et le 20 mai 2009, avait suscité seulement 26 observations (dont 9 à la Possonnière). Les remarques formulées par des particuliers ou des représentants d'association portaient sur le manque d'ambition du projet (notamment sur la question du piégeage des sédiments) et sur le réemploi des $24500 \mathrm{~m}^{3}$ d'enrochements extraits du lit du fleuve, finalement exportés en carrière pour la majeure partie. Seul un pêcheur professionnel contestait le remodelage en invoquant notamment le risque de modification des terroirs de pêche. Deux structures de logistique se sont inquiétées du maintien des conditions de navigabilité et du potentiel de transport fluvial. Enfin une seule association locale de sauvegarde de l'environnement et du patrimoine (La Possonnière) a émis de très fortes réserves à la modification des épis pour des raisons écologiques et techniques. Lors de l'enquête menée après les travaux, la question "Que pensez-vous des travaux? » a fait apparaître plusieurs groupes d'opinions. Le premier est constitué de personnes qui connaissent toutes les raisons du remodelage, tout au moins l'objectif de relèvement de la ligne d'eau. Ceux-là considèrent l'initiative intéressante mais attendent les résultats du suivi pour en juger. Le second groupe concerne les personnes qui ne se prononcent pas car ils ne connaissent pas la raison des travaux. Le troisième est plutôt en désaccord avec l'expérimentation, considérant que le problème est mal traité. Ils mettent en avant le déséquilibre du fleuve engendré par la destruction des seuils rocheux dans les années 1970 et l'extraction sablière, qui ont entraîné une baisse du niveau d'eau à l'étiage entre 1,5 et 4 mètres. Selon eux, le projet n'est pas assez ambitieux au regard de l'ampleur du problème, le remodelage des épis n'entraînant qu'une baisse proportionnelle à leur responsabilité dans l'abaissement de la ligne d'eau. On peut enfin ajouter ceux qui considèrent ce projet inutile car inefficace : ils mettent en doute la sédimentation du sable dans le fond du chenal, par manque de rugosité du fond du lit : le sable ne serait pas remobilisé et irait jusqu'à l'Océan par le biais de l'estuaire qui «avale» tous les matériaux de l'amont.

\section{Conclusion}

L'idée de la Loire chenalisée, moteur d'un développement économique régional (voire national) résiste à la forte mobilisation locale et à son échec pratique patent. En effet, jamais l'entretien, les modifications successives du tracé du chenal n'ont permis le décollage d'un trafic fluvial important. Alors que le tournant environnemental (1990) instaure un nouveau paradigme de gestion du fleuve (adapter les usages au fleuve et non modeler le fleuve pour des usages inadaptés), les structures de gestion du chenal sont maintenues et les programmes lourds de rechargement des épis se poursuivent jusqu'au milieu des années 1990. Aujourd'hui encore, l'utilisation du chenal pour la navigation commerciale n'est pas encore totalement abandonnée (VNF, 2009).

Sur le plan paysager, les épis apparaissent dès leur aménagement comme des objets ambivalents. Ils symbolisent pour les partisans de la Loire navigable, l'art des ingénieurs et le progrès tandis que les opposants les associent à des marques de blessures infligées au fleuve. Les épis sont d'abord très visibles au moment de leur installation puis deviennent rapidement plus discrets avec le stockage du sable. Mais de nouveau l'enfoncement du lit du fleuve leur redonne une place importante dans le paysage à l'étiage. Les rechargements réguliers entre 1960 et le milieu des années 1990 modifient leur aspect (enrochements). Ces aménagements transverses ont contribué à modifier le rapport au fleuve. Requalifiés par les usages locaux, ils sont devenus des éléments paysagers ordinaires. Quelques campagnes de promotion touristiques ont contribué à une esthétisation de ces infrastructures. Mais si l'on peut identifier des valeurs d'attachement local à ces ouvrages, la patrimonialité de l'ensemble des épis n'a pas été à ce jour démontrée et bien caractérisée. Si l'on reprend la proposition de C. Larrère et R. Larrère (1997) sur l'analyse des représentations paysagères, on peut dire que le regard savant dominant, informé par la science, qui tend à déprécier les épis du fait de leur impact sur l'écologie du fleuve, cohabite avec le regard initié des habitants, usagers des lieux. Cette géohistoire des épis et l'analyse des représentations actuelles confirme l'intérêt de ne pas assimiler héritage et patrimoine pour distinguer parmi les objets créés au sein des anthroposystèmes (Burnouf et al., 2003) ceux qui sont requalifiés par 
certains groupes sociaux dans leurs propres temporalités et stratégies.

\section{Remerciements}

Cette recherche, issue du programme PATRA (2010-2012, dirigé par Hervé Davodeau) a été financée dans le cadre du Plan Loire Grandeur Nature et a bénéficiée du soutien du FEDER. Nous remercions l'ensemble de l'équipe de l'EPL pour leur accompagnement dans le déroulement de notre projet. Nous remercions également les personnels de la DDTM et de VNF qui nous ont favorisé l'accès à certaines données. Enfin, nous remercions le CLD et son président Monsieur Jacques Birgand, en particulier pour le prêt du cliché de l'île Monsieur.

\section{Bibliographie}

ACT Consultants, 2009. Étude de développement des possibilités du fret sur la Loire, rapport final. VNF, Paris, 199 p.

Baron-Yelles N., 2006. L'inscription du Val de Loire au patrimoine Mondial de l'UNESCO. Réflexions sur les implications institutionnelles d'un nouveau type de protection de la nature, BAGF - Géographies, Vol. 83, n 3, p. 381-393.

Barraud R. Carcaud N., Davodeau H., Montembault D., 2010. Quel tableau géographique des paysages ligériens?, Géosciences, revue du BRGM, nº 12, p. 112-122.

Barron L., 1888. La Loire, Paris, Laurens, 420 p

Bigando E., 2008. Les paysages ordinaires d'Artigueloutan, rapport pour la communauté d'Agglomération Pau Pyrénées, Pau, UMR SET 5603 CNRS, 31 p.

Bonin S., 200I, Paysages et représentations dans les guides touristiques. La Loire dans la collection des guides Joanne/ guides bleus (1856 à nos jours), L'espace géographique, t. 30, $\mathrm{n}^{\circ} 2$, p. $111-126$.

Burnouf J., Carcaud N., Garcin E., 2003. La Loire entre mythes et réalités, 303, Arts, recherches et créations, La Loire, $n^{\circ} 75$, p. 13-17.

Chapplain L., 1840. Le conducteur de l'étranger à Clisson et sur les bords de Sèvre, aux bains de mer, et de Nantes à Nort sur les rives de l'Erdre, Nantes, Sebire, 51 p.

Charrier P., 1997. La vallée de la Loire des Ponts-de-Cé à Bellevue (entre Anjou et Pays Nantais), évolutions et sensibilités, Thèse de géographie, université de Nantes, 376 p.

Charrier P., 2000. Entre Anjou et Pays Nantais, interventions humaines et transformations hydro-morphologiques en Loire armoricaine (1750-1960). Annales de Géographie, 109, p. 115-131.
Cayla P., 2007. Valorisation patrimoniale de la Basse-Loire angevine et armoricaine [en ligne] : [http://www.terresdeloire. net/paysages/basse_loire/theme1/texte_basse_loire.htm].

Coyaud L.-M., 2003. Paysages culturels du Val de Loire, 303, Arts, recherches et créations, La Loire, $\mathrm{n}^{\circ}$ 75, p. 7-11.

D'Andigné A., 1928. Essai sur la Loire navigable, Société d'éditions géographiques, maritimes et coloniales, Paris, 402 p.

Dion R., 1961. Histoire des levées de la Loire, Paris, Chez l'auteur, $312 \mathrm{p}$.

Droz Y., Mieville-Ott V., Forney J., Spichiger R., 2009. Anthropologie politique du paysage. Valeurs et postures paysagères des montagnes suisses, Paris, Karthala, 170 p.

Grace J., 1974, Lettrines II. José Corti, Paris, 256 p.

Larrère C., Larrère R., 1997. Du bon usage de la nature - Pour une philosophie de l'environnement, Paris, Aubier, 355 p.

Menanteau L., Poissonnier B., 2002, Géoarchéologie de la Loire moyenne, du néolithique à l'âge de bronze, Revue de l'Association pour la Recherche de la Région Ancenienne, $\mathrm{n}^{\circ} 17$, p. 58-77.

Michelin Y., 1998, Des appareils photo jetables au service d'un projet de développement : représentations paysagères et stratégies des acteurs locaux de la montagne thiernoise, Cybergeo : European Journal of Geography [En ligne] : article 65, [http://cybergeo.revues.org/5351].

Pordoy C., 2011 . Valeurs et patrimonialité des représentations paysagères autour des épis de la Loire armoricaine, Mémoire de Master II, Histoire de l'Art, université de Rennes II, 95 p.

Rode S., 2010, De l'aménagement au ménagement des cours d'eau : le bassin de la Loire, miroir de l'évolution des rapports entre aménagement fluvial et environnement, Cybergeo, article 506 [en ligne] : [http://cybergeo.revues.org/23253].

Schulé C.-A., 2000. Les crues inondantes en Anjou du Xix ${ }^{\mathrm{e}}$ au $\mathrm{xx}^{\mathrm{e}}$ siècle. Archives d'Anjou, vol. 4, p. 247-253.

Sсншов M., 1900. La Loire navigable, Communication faite au congrès national des travaux publics, Nantes, M. Schwob et fils, $80 \mathrm{p}$.

TAillade A., 2010. Représentations paysagères et pratiques territoriales à propos des épis de la Loire armoricaine, Mémoire de Master 1 mention Histoire, Géographie et Document Spécialité Paysage, (sous la direction de Jean-Baptiste Humeau), université d'Angers, 105 p.

Tricart J., Bravard J.-P., 1991, L'aménagement des trois plus grands fleuves européens : Rhin, Rhône et Danube. Problèmes et méfaits, Annales de Géographie, t. 100, n 561 562, p. 668-713.

Tougener-Bibliographychard-LAFosse G., 1851. La Loire historique et pittoresque, Tours, ed. Lecesne, 5 tomes.

Vauthier-Vézier A., 2007. L'estuaire et le port, L'identité maritime de Nantes au XIXe siècle, Rennes, PUR, 236 p.

Zimmermann M., 1905. La « Loire navigable», le «projet de Grand Central » et l'avenir du port de Nantes, Annales de géographie, t. $14, \mathrm{n}^{\circ} 73$, p. 84-87. 